Original Research Paper

\title{
A Weighted Voting Deep Learning Approach for Plant Disease Classification
}

\author{
${ }^{1}$ Assia Ennouni, ${ }^{1}$ Noura Ouled Sihamman, ${ }^{1} \mathrm{My}$ Abdelouahed Sabri and ${ }^{2}$ Abdellah Aarab \\ ${ }^{1}$ Department of Computer Science, Faculty of Sciences Dhar el Mahraz, University Sidi Mohamed Ben Abdellah, Fez, Morocco \\ ${ }^{2}$ Department of Physique, Faculty of Sciences Dhar el Mahraz, University Sidi Mohamed Ben Abdellah, Fez, Morocco
}

\section{Article history}

Received: $14-08-2021$

Revised: 05-11-2021

Accepted: 09-10-2021

Corresponding Author:

Assia Ennouni

Department of Computer

Science, Faculty of Sciences

Dhar el Mahraz, University Sidi

Mohamed Ben Abdellah, Fez,

Morocco

Emai: ennouni.assia@gmail.com

\begin{abstract}
Plant destruction is usually caused by plant diseases. Without early and reliable detection, it can affect the plants and may eventually cause permanent losses, especially for inexperienced farmers. Therefore, intelligence in agriculture is becoming more and more required. Thereafter early diagnosis and classification are crucial and a very challenging research field in the agriculture sector for its treatment. In this context, many solutions have been proposed. Deep learning has been highly successful and hardly applicable in this problem. However, through pass survey analysis, we notice that there are a few studies in DL for disease classification problems but the precision and outcomes of different traditional DL methods may vary and give a less score for classification. The proposed approach is based on a weighted combination of five deep learning architectures. The weight of each DL architecture is calculated based on its performance using genetic algorithms. The results of the proposed approach are evaluated on the publicly available Plant Village (PV) dataset. It is found that using the Deep Learning weighted voting method gives higher classification accuracy compared to the results obtained using each DL architecture separately and also compared to recent approaches in literature, which allowed us to correctly identify the leaves and to improve the classification accuracy rate to $99.21 \%$.
\end{abstract}

Keywords: Smart Agriculture, Deep Learning, Ensemble Learning, Weighted Voting, Plant Disease Classification

\section{Introduction}

It is well-known that plant diseases cause appreciable crop losses all over the world and they have a vandal effect on agricultural products. Plant diseases are the first wasting of agricultural progression and have an immediate impact on the quality and quantity of plant foods. If they are not controlled, detected and treated in time, there will be a rise in food insecurity (Fina et al., 2013). Morocco such like any African countries depends sorely on agriculture. Recently, FAO declares a considerable increase due to many causes such as climate change.... but plant disease detection remains the most important factor in this problem. To address the considerable problem of plant disease diagnosis and help farmers in their decisions, artificial intelligent approaches for agricultural image analysis can be utilized to develop a performant classification system that can detect plant diseases using only images of leaves (Ennouni et al., 2021a). Deep learning approachs have been used for medical image classification (Filali et al., 2020) In this trend, deep learning applications are proposed and implemented to agricultural imaging tasks are thoroughly examined (Litjens et al., 2017; Biswas et al., 2019; Abdelhafiz et al., 2019; Lundervold et al., 2019).

In this study, a novel method for detecting and classifying plant diseases is proposed based on the Ensemble Learning (EL) technique. Our proposed approach aggregates five deep learning architectures naming VGG16, AlexNet, CNN, Inceptionv3 and mobileNet in an ensemble learning scheme using a weighted voting mechanism. The optimal weights are assigned by the genetic algorithm to achieve the optimal ones and improve the classification accuracy. The performance of the suggested approach is assessed on the publicly available Plant Village dataset (PV).

\section{Related Work}

Plant diseases are a worldwide agricultural problem in precision agriculture. It is considered the one kind of natural disaster that attacks the normal growth process of plants and this problem causes plant death in most cases 
during the whole growth process of plants from seed development to seedling and seedling growth. The various diseases that can affect plants can be categorized into three types: Fungal, bacterial, or viral (El-Sayed et al., 2020; Vijai et al., 2020).

\section{Viral Diseases}

This type is identified by these classic symptoms mosaic patterns, yellowing, stripes and leaf rolling as you have shown in Fig. 1. Viral diseases are diffused to plants principally by insects or worms.

\section{Fungal Diseases}

The symptoms of these diseases are leaf rust especially for corn, stem rust, white mold (Sclerotinia). They considered damaging to plants but not with great risk. Fungi, mold, mildew and others cause these diseases. Figure 2 shows an instance of a plant affected by Sclerotinia fungus.

\section{Bacterial Diseases}

They can be detected by the attendance of rot, scab, scorch, wilt and leaf spots. Plant infections of bacterial have almost similar symptoms to fungal diseases. These types of diseases, if not treated in time, can cause serious and disastrous diseases (Park et al., 2017). An example of bacterial disease is shown in Fig. 3.

In general, plant disease symptoms are hard to discover in time as result early plant diagnosis by experts is required but expensive (Mathews, 2010). There are many directed studies on machine learning-based plant diagnosis in recent years (Barbedo, 2013; Huang, 2007; Phadikar and Sil, 2008) to improve diagnosis results. In (Barbedo, 2013) authors used a multi-layer perceptronbased recognition method to detect bacterial soft rot, bacterial brown spot and phytophthora black rot appearing on orchids, this study reported an average classification accuracy of $89.6 \%$. Others (Huang, 2007) suggest a method based on self-organized; it achieved over $70 \%$ accuracy in distinguishing between rice blast and brown spot appearing on rice leaves. In (Phadikar and Sil, 2008) researchers applied SVM for distinguishing brown spots, downy mildew and angular leaf spot on cucumbers and reached an accuracy of $83.3 \%$. In (Zhang et al., 2018) analyzed nutrient deficiency in tomatoes utilizing k-nearest neighbor clustering based on the leaf, the authors extract two descriptors color and texture. However, it is quite difficult to handle these techniques for detecting different plant diseases (feature representation). That means all these proposed techniques are confronted with several difficulties, concerning the detection of regions of interest for succeeding processing (Xu et al., 2011). The effects of deep learning architectures are noticeably greater than those of the traditional machine learning methods in the most application process. Deep learning models are capable to learn feature representations from raw data and are not dependent on feature engineering that many works have underlined the importance of deep learning in agricultural tasks comparing with the traditional ML techniques for improving the quality and safety of nutrition.

In recent years, we notice an immense interest in deep learning in various fields (LeCun et al., 2015). Deep Learning (DL) architectures, including Convolutional Neural Networks (CNN), has arisen as the most encouraging approaches given their ability to learn reliable and discriminative visual characteristics. Many studies have emphasized and proved the importance of deep learning in agriculture as compared to ML methods, for instance, the famous DL model named Google Net outperformed the ML techniques including Support Vector Machine and Random Forest algorithms for the classification of disease in tomato leaves (Ennouni et al., 2021a). In this section, we present recent researches that reveal the entire penetration of deep learning.

Authors in these researchers present many state-of-the-art architectures containing MobileNet (Howard et al., 2020), Visual Geometry Group (VGG) (Brahimi et al., 2017; Ferentinos et al., 2008; Mohanty et al., 2016; Zhang et al., 2018; Oppenheim et al., 2019), AlexNet (Howard et al., 2020; Brahimi et al., 2017; Ferentinos et al., 2008; Fuentes et al., 2017; Mohanty et al., 2016; Türkoğlu et al., 2019; Zhang et al., 2018), ResNet (Fuentes et al., 2017; Türkoğlu et al., 2019; Zhang et al., 2018; Too et al., 2019), Inception-v4 and DenseNet (Too et al., 2019), got promising results for the classification of plant leaf disease. This table displays the popular and successful architectures including classic and modern architectures. As you see in this table we identify for all architecture; the number of parameters, the authors, the year and the depth.

Table 1: The most successful CNN architectures

\begin{tabular}{lllll}
\hline Architectures & References & Year & Nb of params & Depth \\
\hline LeNet-5 & LeCun et al. & 1998 & 60,000 & 5 \\
AlexNet & Krizhevsky et al. & 2012 & 60 million & 138 million \\
VGG & Simonyan and Zisserman & 2014 & 4 million & 19 \\
GoogleNet & Szegedy et al. & 2015 & 23 million & 22 \\
Inception V3 & Szegedy et al. & 2015 & 35 million & 159 \\
Inception V4 & Szegedy et al. & 2016 & 25 million & 70 \\
ResNet & He et al. & 2016 & 4.2 million & 152 \\
MobileNetV1 & Sandler et al. & 2018 & 3.47 million & 28 \\
MobileNetV2 & Mark et al. & 2018 & & 53 \\
\hline
\end{tabular}




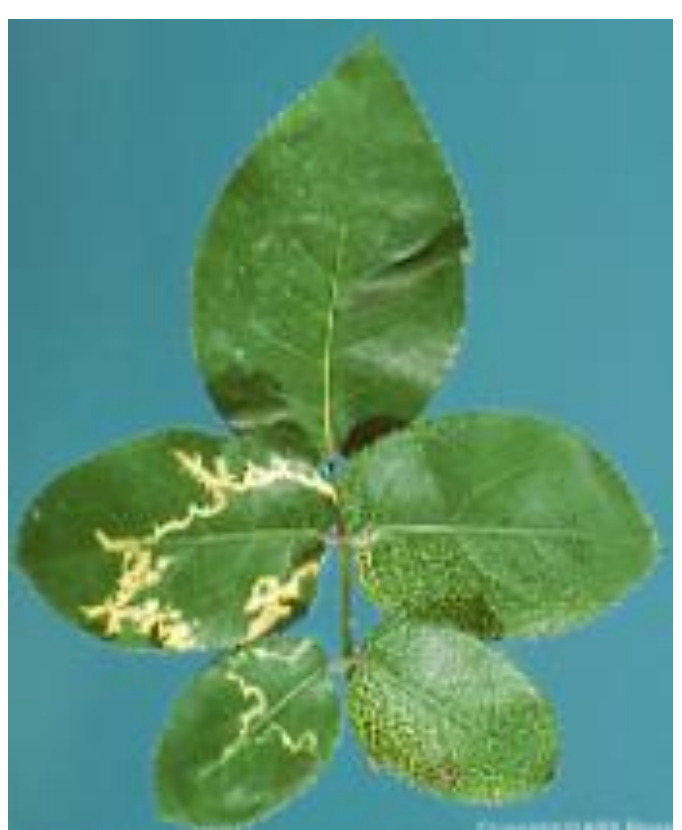

Fig. 1: Rose mosaic virus leaves
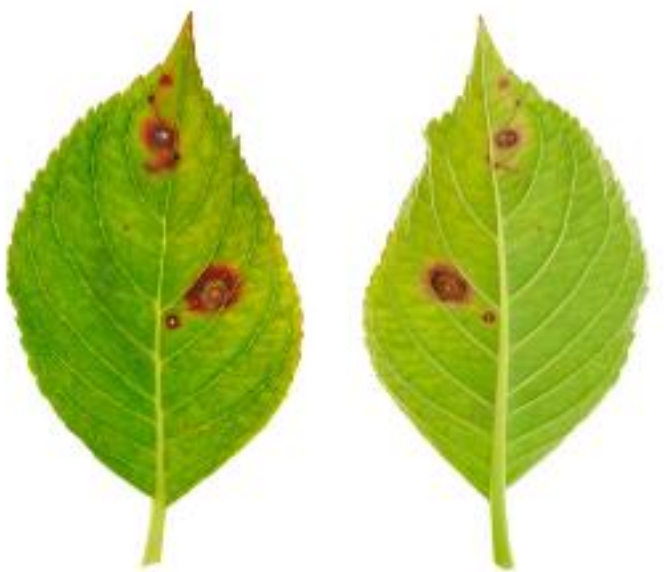

Fig. 2: Sclerotinia Infected by Soybeans

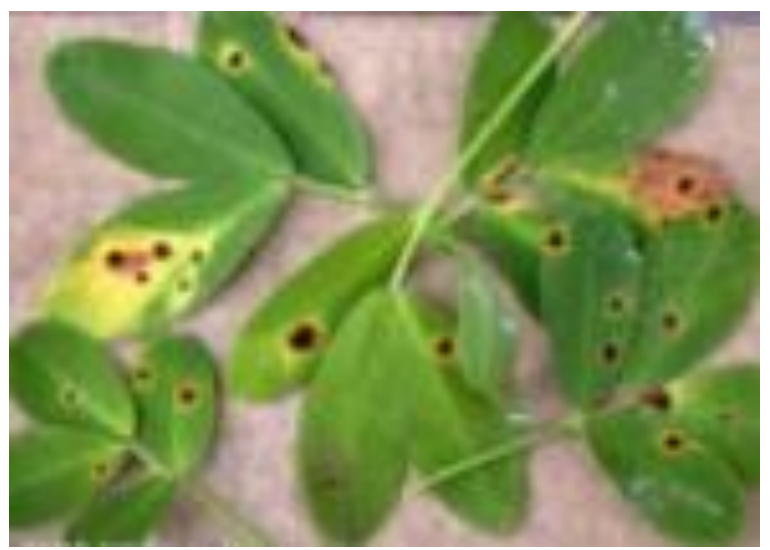

Fig. 3: Bacterial blight of peas leaves
As you see before, the number of architectures and algorithms that are used in deep learning is varied and wide. In this section, we explore the famous deep learning architectures and we take a look in more detail at each of these to give you a deeper understanding of applying these approaches in practice.

\section{LeNet-5}

Starting with LeNet-5 (LeCun et al., 1989), LeNet-5 $\mathrm{CNN}$ is one of the earliest models developed by LeCun et al. (1998); in the research work, Gradient-based Learning Applied to Document Recognition. It is used to identify handwritten and machine-printed characters. This architecture is simple in that It is popular for image classification. It has 5 layers with learnable parameters and 3 convolution layers, two average pooling layers and 2 fully connected layers with a softmax classifier. The presented Fig. 4 depicts the architecture of LeNet5 in the original paper.

\section{ALexNet}

Krizhevsky et al. (2012) won the Image Net ILSVRC 2012 by developing a very similar architecture (Krizhevsky et al., 2012) as Le Net by LeCun et al. (1989). Their architecture reducing the top-5 error from $26 \%$ to $15.3 \%$ and considered as a leading architecture with a total of eight layers with learnable parameters. The fundamental highlights of this proposed architecture are these: First, used ReLU instead of tanh to add nonlinearity. Second, prevented their model from overfitting by using dropout instead of regularization. Finally and to reduce the size of the network they used overlap polling. It has five layers with a combination of max pooling followed by 3 fully connected layers. They use ReLU activation in each of these layers except the output layer. AlexNet architecture is shown below in Fig. 5.

\section{$V G G$}

While the AlexNet model focused on smaller windows sizes and strides in the first convolutional layer. VGG come out in 2014 based on depth (Simonyan and Zisserman, 2014). Simonyan introduced the popular convolutional neural network architecture called VGG in their work intituled 'Very Deep Convolutional Networks for Large-Scale Image Recognition' VGG concentered on another very important aspect of CNNs which is depth. Its work was focused on an analysis of how to increase the depth of the network, using smaller filters $(3 * 3)$ with more depth instead of having large filters. The best results can be achieved by pushing the depth to 16-19 weights layers. The proposed model has achieved $92.7 \%$ top-5 test accuracy in Image Net. Noted that the VGG model supports up to 19 layers. The network architecture is provided in the following Fig. 6. 
In the same context, authors Szegedy et al. (2015) proposed a new type of deep convolutional neural network named Inception which is called Google Le Net or Inception in their work entitled "Going deeper with convolutions" (Szegedy et al., 2015) for ILSVRC14. The focal point of this architecture is the improved utilization of the computing resources inside the network. They increased the depth and width of the network while keeping the computational budget constant. It is essentially a Convolutional Neural Network (CNN) that is 27 layers deep. The basic implementation of this model was called Google Net. Later publications were then named Inception VX where $\mathrm{X}$ refers to the number of the version. The presented Fig. 7 depicts the architecture of inception.

\section{ResNet}

Now, we will discuss the Residual Network Known as ResNet by Kaiming He et al. (2016) which is one of the most robust deep neural networks which has obtained excellent performance results in the ILSVRC 2015 classification challenge. Every layer of a ResNet (Zhang et al., 2018) is composed of several blocks. This is because when ResNets go deeper, they normally do it by increasing the number of operations within a block, but the number of total layers remains the same. The principal idea of ResNet is (Sibiya and Sumbwanyambe, 2019) presenting a so-named "identity shortcut connection" that skips one or more layers to deal vanishing gradient problem. Instead of hoping every few stacked layers directly fit a desired underlying mapping, residual nets let these layers fit a residual mapping. Figure 8 illustrates this architecture.

\section{MobileNet}

Mobile Net architecture [31] introduced by Howard et al. (2017) in Mobile Nets: Efficient Convolutional Neural Networks for Mobile Vision Applications in 2017. It is a lightweight deep neural network that provides a robust model for embedded and mobile applications (Sahni et al., 2021). It utilizes depth-wise separable convolutions instead of standard convolutions to decrease model size and computation. So we can see, Mobile Net is built on depth wise separable convolutions (Szegedy et al., 2015), except for the first layer. The first layer is a full convolutional layer. The Fig. 9 below shows the Mobile Net architecture based on depth wise separable filters.

\section{Plant Disease Classification Based Ensemble Learning Mechanisms}

Ensemble learning is a machine learning paradigm where multiple learners are trained to solve the same problem. It has been demonstrated that combining multiple classifiers can be more effective than any single one (Onan et al., 2016). From a technical standpoint, ensemble learning is primarily implemented in two steps: Training weak classifiers and selectively combining the member classifiers into a stronger classifier. Based on this source (Xiao et al., 2018), it is conceivable to combine the performance of a set of classification architectures to obtain a single robust classification model each architecture has strengths and weaknesses. Usually, the members of an ensemble are built in two ways. One is to apply a single learning algorithm and the other is to use different learning algorithms over a dataset (Chen et al., 2014). Then, the base classifiers are combined to form a decision classifier. Generally, to get a good ensemble, the base learners should be as more accurate as possible and as more diverse as possible. So how to choose an ensemble of some accurate and diverse base learners is a focus of concern of many researchers. In recent years, more and more researchers are concerned with ensemble learning (Zhou et al., 2009). The major principles of ensemble learning for analysis could be classified into these methods, bagging, boosting, stacking and voting described as follows.

Bagging is the most basic and simplest method; it involves two steps; bootstrapping and aggregation, in which a single training algorithm is applied to different subsets of training data, with subset sampling performed with replacement (bootstrap). Once the algorithm has been trained across all subsets, bagging predicts by aggregating all of the predictions made by the algorithm across all subset. Because the sampling is done by bootstrapping, the bagging method works so well because of the diversity in the training data. It reduces variance while not increasing bias (Breiman, 1996; Petropoulos et al., 2018).

Boosting: This is a machine learning algorithm in which the weights of the classifiers are iteratively adjusted. The principle underlying the boosting approach was based on the importance of more complex models. The classification piqued people's interest in the amount of weight set in each model of the training set (Wang and Ma, 2011). By decreasing the distribution and combination of weak classifiers, the AdaBoost algorithm developed the weight of each case by training a set of the same and different weak classifiers. Finally, the AdaBoost algorithm was used to construct the final decision using weighted majority voting.

Stacking is another well-known ensemble learning technique for achieving the best possible outcome which frequently considers heterogeneous weak learners, learns them in parallel and combines them by training a meta-model to output a prediction based on the predictions of the different weak models. The stacking architecture set (Wolpert, 2011) was a two-level structure made up of level-0 (base-level) classifiers and level-1 (meta-level) classifiers. The training dataset was used to train the base-level classifiers, which were then used to construct the prediction. Metadata was later used to train a metaclassifier to recognize the output of the base-level classifier as the class label. Stacking was generally used to combine the developed model with the various classifiers. These classifiers were combined in various predictions to form the final resolution. 


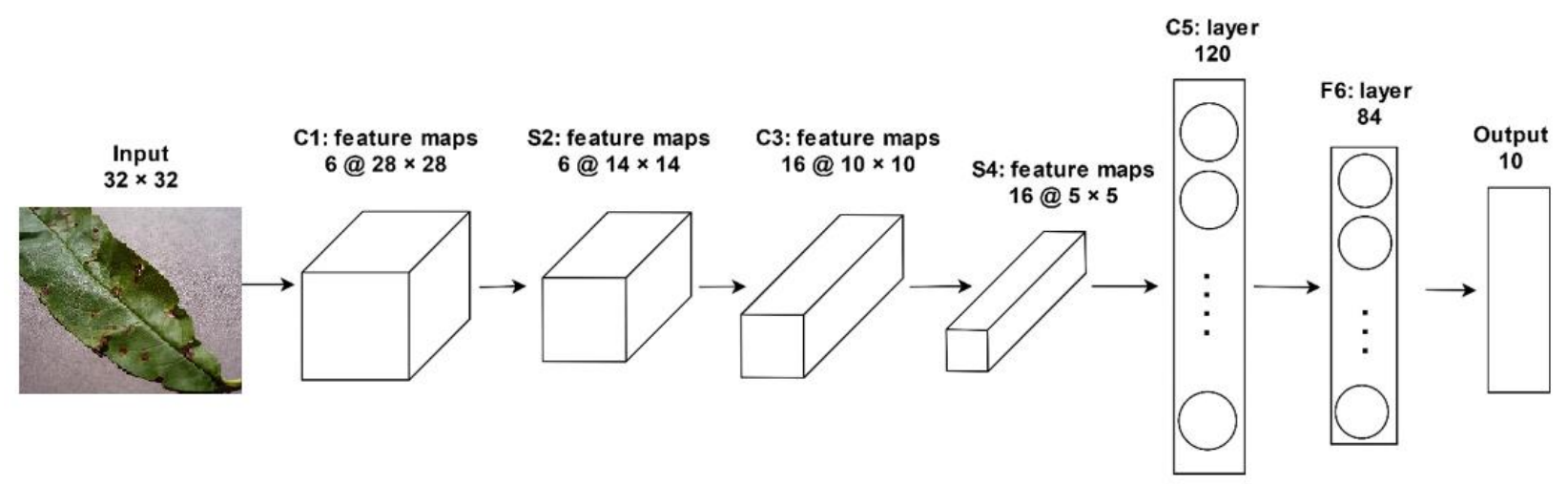

Fig. 4: LeNet-5 architecture
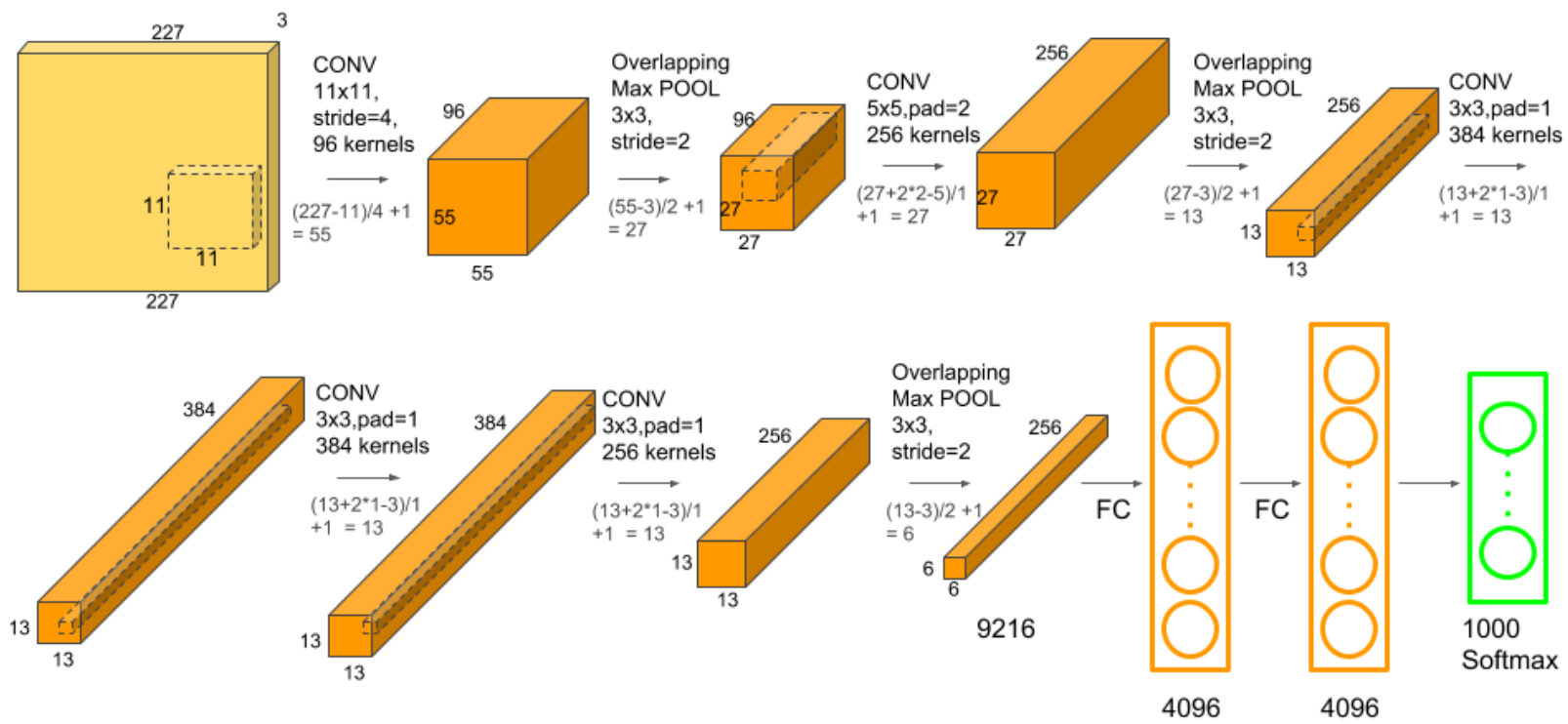

Fig. 5: Alex Net Architecture

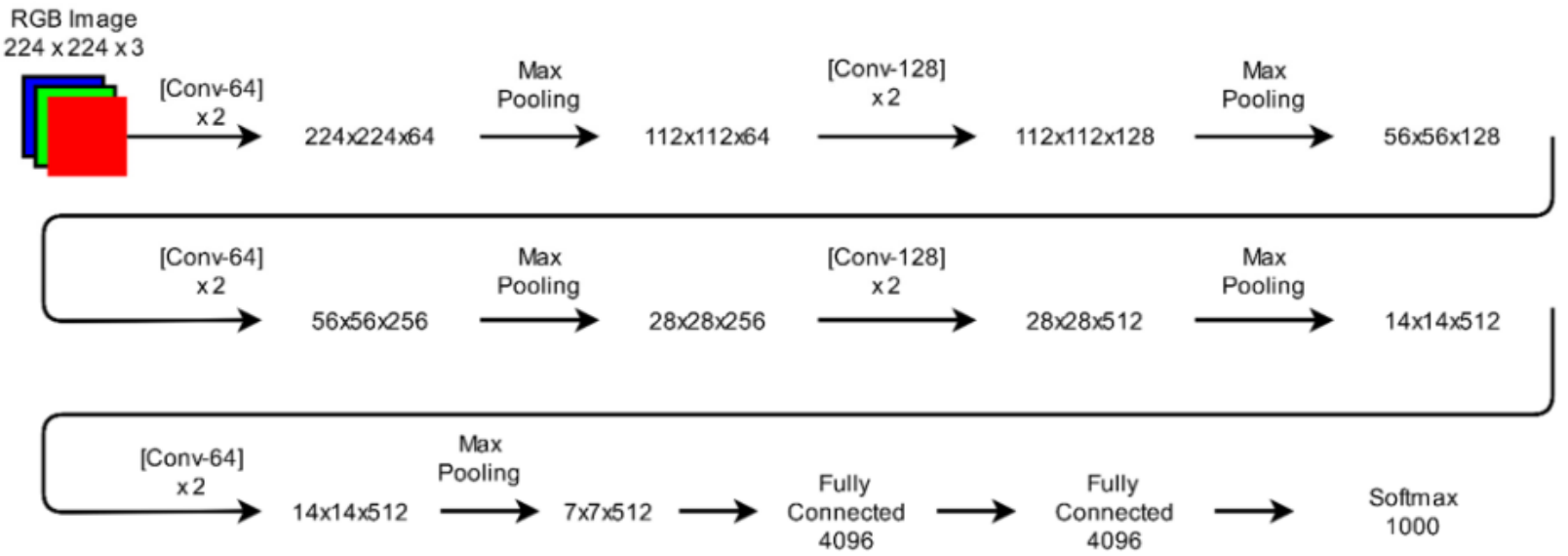

Fig. 6: VGG16 Architecture 


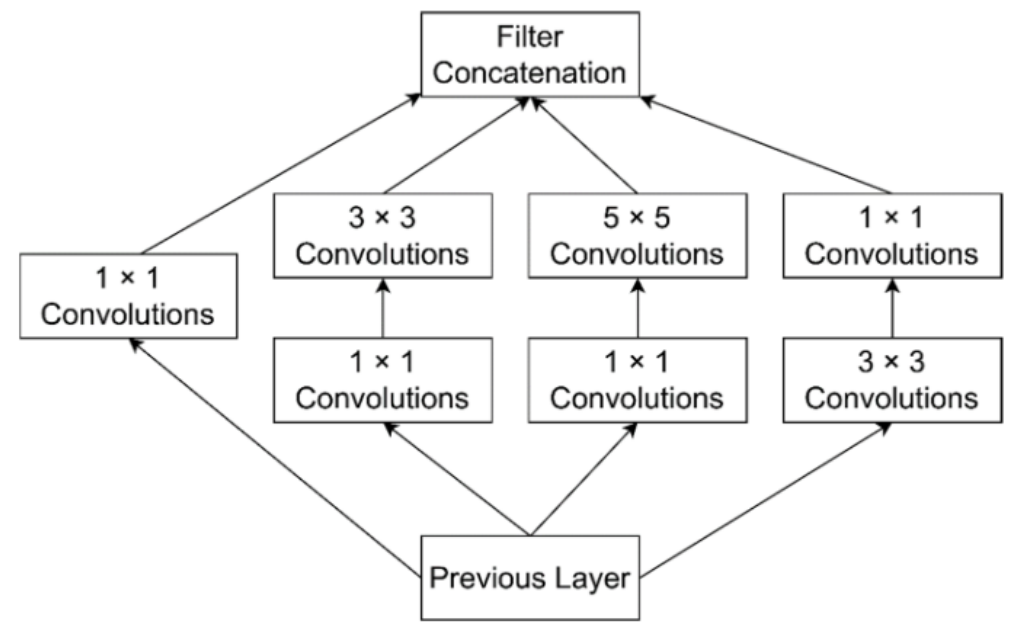

Fig. 7: Inception module with dimensionality reduction

Voting method used to make decisions by selecting only one of several alternatives. Voting depended on the class predicted with the majority voting. Moreover, voting was the most utilized in the ensemble methods. Generally, the voting method consisted of unweighted voting and weighted voting. Unweighted voting consisted of simple voting and majority voting, whereas weighted voting included simple weighted voting (Kuncheva, 2014). It supports these types of voting.

Hard voting or Majority-Based Voting Mechanism includes summing the predictions for each class label and predicting the class label with the most votes that means the final class prediction is made by the majority vote which the estimator selects the class that occurs most frequently among the base models (Delgado and Ishii, 1999; Kang et al., 2020).

$$
\tilde{y}=\operatorname{Mode}\left\{C_{1}(x), C_{2}(x), \ldots, C_{n}(x)\right\}
$$

Weighted voting was simple voting that was considered the suitable method for all classifiers with equivalent performance. However, the base classifier was practically carried out with different weights for defining the weight. Thus, weight voting was designed to define the weight for constructing a strong classifier (Tyagi et al., 2021). We can compute a weighted majority vote by associating a weight $w j$ to the classifier $C j$ :

$$
\tilde{y}=\arg \max \sum_{j=0}^{m} W_{j} C_{j}(x)
$$

Soft voting has the same principle as earlier but is expressed in terms of probabilities. That means the final output is predicted based on the predicted probabilities $p$ for the classifiers (Du and Swamy, 2019). For each class label and predicting the class label with the largest probability. In each case below, the probability of class labels assigned by the classifier $\mathrm{C}$ to input $\mathrm{x}$ is defined as (Cao et al., 2015):
Average of Probabilities Voting:

$$
\tilde{y}=\text { Average }\left\{C_{1}(x), C_{2}(x), \ldots, C_{n}(x)\right\}
$$

Product of probabilities voting:

$$
\tilde{y}=P R O D\left\{C_{1}(x), C_{2}(x), \ldots, C_{n}(x)\right\}
$$

Minimum of probabilities voting:

$$
\tilde{y}=\operatorname{MIN}\left\{C_{1}(x), C_{2}(x), \ldots, C_{n}(x)\right\}
$$

Maximum of probabilities voting:

$$
\tilde{y}=\operatorname{MAX}\left\{C_{1}(x), C_{2}(x), \ldots, C_{n}(x)\right\}
$$

\section{Proposed Methods}

In this proposed approach, we present a new and powerful approach for plant disease classification based on weighted voting approach using Deep learning models as ensemble of base classifiers. The proposed approach is based on a weighted combination of five deep learning architectures. The weight of each DL architecture is calculated based on its performance using genetic algorithms. The optimal weights of each of the 5 architectures is optimized by the Genetic Algorithm (GA). The overall workflow of the proposed method has been depicted in this Fig. 10.

As we can see clearly, after applying the five abovementioned architectures, using the different voting mechanisms to improve the classification results. Each of these architectures' classification results is calculated for each test instance and the final results are predicted based on the weighted majority voting results. Details of the voting mechanism used are given in the above sub-sections. 
We summarize the mainly steps in this the following descriptions:

Step 1: First, before applying the ensemble learning on the Dataset, we perform a fine-tuning of each of the 5 Deep learning algorithms to be used in order to define the hyper parameters to be used.

Step 2: Applying the Global Average Pooling (GAP) layer reduces each feature map to a single number by simply taking the average of all values and minimizes overfitting by reducing the total number of parameters in all models.

Step 3: We proceed to combine the $5 \mathrm{DL}$ algorithms. The weights of each of the 5 algorithms are optimized using the genetic algorithms.

Step 4: The final prediction is obtained by weighted voting using the optimal weights obtained by step 3

Now we select our weights parameter. Here, we will use Genetic algorithm approach to find the optimal weights for each classifier to increase the prediction accuracy.

\section{Genetic Algorithm for Tunning the Weight}

Now we select our weights parameters. Here, we will use Genetic algorithm approach to find the optimal weights for each classifier to increase the prediction accuracy.

The principal steps of the used genetic algorithm (Yuan et al., 2012) are: (1) Initialization by producing a collection of many individuals and each individual denotes the weight of each classifier. (2) Fitness: The GA calculates the fitness of individuals based on some objective evaluation function, through learning the survival probability of individuals in the following evolution. (3) Selection: Using random or specific population rules to participate in cross and mutation. We select a certain number of excellent individuals with "more fitness" (4) frequently great individuals are usually of high fitness, that is, better.

\section{Experimental Results}

\section{Dataset}

In our work, each single deep learning model and the ensemble learning models were trained on a publicly available dataset called plant village (Hughes and Salathé, 2015). This dataset is one of the most used for the evaluation of plant disease classification which contains 54,306 images in total classified into 38 subsets. Some of them are displayed in the Fig. 11. It covers healthy and diseased leaves to their categories. For making it appropriate for the initial values of the trained models. The size of images was modified to 224 $\times 224 \times 3$ and normalization was measured by dividing the values of pixel by 255. To avoid the overfitting problem, we divide our dataset into 3 categories training 70, 20\% for validation and $10 \%$ for testing. The dataset is provided also by the Ground of Truth (GT) that will help us to evaluate the approach. Table 2 listed a detailed description of the used dataset and also the abbreviation of each class used in the next subsections.

\section{B. Performance Measures}

In this study, we evaluate the performance of our proposed approach by calculating common classification indicators. we will consider the statistics of correct classification (also known as true positives) which means the number of classes that the classifier accurately classifies, misclassification (also known as false negatives) which is the number of classes that are mistakenly identified, False Positive signify the number of cases which are inaccurately marked as such. TRUE Negative (TN) is the number of examples that are not in such disease. These measures are accuracy, sensitivity and specificity. For precision is the ratio of correctly predicted positive observations to the total predicted positive observations call is the ratio of correctly predicted positive observations to all the observations in the actual class. F1-Score is the weighted average of Precision and Recall as defined in Equations.

$$
\begin{aligned}
& \text { precision }=\frac{\sum_{i=1}^{n} \text { precision }_{i}}{n} \\
& \text { where precision }_{i}=\frac{T P_{i}}{T P_{i}+F P_{i}} \\
& \text { recall }=\frac{\sum_{i=1}^{n} \text { recall }_{i}}{n} \\
& \text { where recall }_{i}=\frac{T P_{i}}{T P_{i}+F N_{i}} \\
& \text { accuracy }=\frac{\sum_{i=1}^{n} \text { accuracy }_{i}}{n} \\
& \text { where accuracy }_{i}=\frac{T P_{i}+F P_{i}}{T P_{i}+T N_{i}+F P_{i}+F N_{i}}
\end{aligned}
$$

Where:

$n=$ The number of classes, $\mathrm{i}$ the current class

$T P=$ The True Positives

$F P=$ The False Positives

$F P=$ The False Positives

$F N=$ The False Negatives

\section{Simulations Results}

We used python language for programming ensemble learning architectures based on the three different voting mechanisms to the simplicity and availability of very useful DL frameworks and libraries. For instance, we used Keras and Tensor Flow to build all these architectures. All 
our experiments were carried out on a Graphical Processing Unit (NVIDIA Quadro K2200) having the specifications: 32GB memory, 640 CUDA cores, 1045 $\mathrm{MHz}$ core clock and $80 \mathrm{~GB} / \mathrm{sec}$ memory bandwidth.

For the experiments, we use 10-fold cross-validations for different architecture. For learning rate, we use 0.00001 and Adam method [41] for adaptive learning and cross-entropy as loss function. For Plant village, we trained all five architectures for all plants images and set the epochs into 100 .

Table 3 summarizes the studied approaches that use DL models for plant diseases classification, where the results are based on accuracy, as well as the metrics that they used and the final results are presented as follows.

After examining these results, we notice all of the architectures showed a significant performance, starting with accuracy, Inception and VGG16 had the lower values with 91 and $92 \%$ respectively. Followed by the common result founded with AlexNet, $\mathrm{CNN}$ and Mobile Net with $95 \%$. The best recall representing by Mobil Net provides almost excellent Classification results which achieved 97\%. On the other hand, in the Precision indicator, Alex Net obtained the lower result with $86 \%$ followed by VGG16, InceptionV3 and Mobile Net with $89 \%$ to both and $94 \%$, respectively and the highest value was obtained by CNN with $95 \%$. In addition, we consider another metric which is processing time, we remark that Alex Net gets the best performance by taking the shorter time.

To improve the accuracy results for these models we implemented three voting mechanism-based ensemble learning classifications; Hard voting, weighted voting and soft voting. The last one includes diverse voting schemes that were tested, minimum probabilities, maximum probabilities, the product of probabilities and the average of probabilities. All architectures are combined and trained using all mechanism voting. So the accuracy scores related to these comparative analyses have been given in Table 4 . Notice that ELA abbreviation means Ensemble Learning Architectures.

As seen in Table 4 and Fig. 12, the best performance was achieved using the weighted voting mechanism comparing with the other mechanisms voting for the plant village dataset. On the other hand, hard voting obtained the lowest performance compared to others. That means our proposed approach considers more information by combining the five architectures in the final prediction. So our goal was to find the more suitable voting mechanism by combining five architectures in ensemble learning to enhance the accuracy of proposed models and classify the leaves diseases in their classes. However, the proposed Ensemble Learning approaches have significantly improved the classification results and especially using the weighted voting technique with a value equal to $99.21 \%$. We can say that the use of the vote of the 5 architectures allowed us to have the best classification rate as we see in Fig. 13.
Furthermore, Table 5 and 6 show the confusion matrix with the best output based on the performance measures, which are the weighted voting mechanism-based combined five models. Depending on the results, it is simple to visually evaluate the performance of the classifier and to determine which classes are highlighted by our approach. The rows are related to the output class, while they are related to the true class. The diagonal cells are associated with the observations that are correctly classified and the off-diagonal cells correspond to the incorrectly classified observations.

Table 1: The most successful CNN architectures

\begin{tabular}{lllll}
\hline Architectures & References & Year & Nb of params & depth \\
\hline LeNet-5 & LeCun et al. & 1998 & 60,000 & 5 \\
AlexNet & Krizhevsky et al. & 2012 & 60 million & 8 \\
VGG & Simonyan et al. & 2014 & 138 million & 19 \\
GoogleNet & Szegedy et al. & 2015 & 4 million & 22 \\
Inception V3 & Szegedy et al. & 2015 & 23 million & 159 \\
Inception V4 & Szegedy et al. & 2016 & 35 million & 70 \\
ResNet & He et al. & 2016 & 25 million & 152 \\
MobileNetV1 & Sandler et al. & 2017 & 4.2 million & 28 \\
MobileNetV2 & Mark et al. & 2018 & 3.47 million & 53 \\
\hline
\end{tabular}

Table 2: Description of plant village dataset

\begin{tabular}{|c|c|c|}
\hline Class name & Abbreviation & $\begin{array}{l}\text { Number } \\
\text { of images }\end{array}$ \\
\hline Apple scab & $\mathrm{AS}$ & 498 \\
\hline Apple black rot & $\mathrm{ABR}$ & 484 \\
\hline Apple cedar apple rust & ACAR & 220 \\
\hline Apple healthy & $\mathrm{AH}$ & 1336 \\
\hline Blueberry healthy & $\mathrm{BH}$ & 1231 \\
\hline Cherry powdery mildew & CPM & 948 \\
\hline Cherry healthy & $\mathrm{CHH}$ & 703 \\
\hline Corn healthy & $\mathrm{CH}$ & 934 \\
\hline Corn Northerm leaf blight & CNLB & 798 \\
\hline Corn common rust & CCR & 954 \\
\hline Corn cercospora leaf gray leaf spot & CCLGLS & 409 \\
\hline Grape blackrot & GB & 884 \\
\hline Grape esca & GE & 1099 \\
\hline Grape leaf blight & GLB & 828 \\
\hline Grape healthy & $\mathrm{GH}$ & 341 \\
\hline Orange citrus greening & OCG & 4361 \\
\hline Peach bacterial spot & PBS & 1819 \\
\hline Peach healthy & $\mathrm{PEH}$ & 280 \\
\hline Pepper bell healthy & $\mathrm{PBH}$ & 1267 \\
\hline Pepper bell Bacterial spot & PBBS & 781 \\
\hline Potato healthy & $\mathrm{PH}$ & 116 \\
\hline Potato Late blight & PLB & 768 \\
\hline Potato Early blight & PEB & 824 \\
\hline Raspberry healthy & RH & 208 \\
\hline Soybean healthy & $\mathrm{SH}$ & 4202 \\
\hline Squash powdery mildew & SPM & 1503 \\
\hline Strawberry healthy & STH & 388 \\
\hline Strawberry leaf scorch & SLS & 931 \\
\hline Tomato Target Spot & TTS & 1136 \\
\hline Tomato Tomato mosaic virus & TTMV & 307 \\
\hline Tomato Tomato Yellow Leaf Curl Virus & TTYLCV & 4312 \\
\hline Tomato Bacterial spot & TBS & 1739 \\
\hline Tomato Early blight & TEB & 839 \\
\hline Tomato healthy & $\mathrm{TH}$ & 1266 \\
\hline Tomato Late blight & TLB & 1560 \\
\hline Tomato Leaf Mold & TLM & 768 \\
\hline Tomato Septoria leaf spot & TSLP & 1456 \\
\hline $\begin{array}{l}\text { Tomato Spider mites Two } \\
\text { spotted spider mite }\end{array}$ & TSMTSSm & 1312 \\
\hline
\end{tabular}


Table 3: Performance measures for every model

\begin{tabular}{|c|c|c|c|c|c|}
\hline Models & Precision & Recall & F Score & Time $(\min )$ & Accuracy \\
\hline VGG16 & 0.89 & 0.96 & 0.96 & 94.95 & $92 \%$ \\
\hline Alex Net & 0.86 & 0.96 & 0.95 & 94.17 & $95 \%$ \\
\hline InceptioV3 & 0.89 & 0.95 & 0.95 & 133.12 & $91 \%$ \\
\hline $\mathrm{CNN}$ & 0.95 & 0.95 & 0.95 & 99.20 & $95 \%$ \\
\hline Mobile Net & 0.94 & 0.97 & 0.94 & 147.73 & $95 \%$ \\
\hline
\end{tabular}

Table 4: Performance measures for combining DL models and voting approaches

\begin{tabular}{lllll}
\hline Models based voting mechnism & Precision & Recall & FScore & Accuracy \\
\hline Hard ELA & 0.9940 & 0.9940 & 0.9940 & $91.1 \%$ \\
SOFT ELA based Average Probabilities & 0.989 & 0.988 & 0.9881 & $98.82 \%$ \\
SOFT ELA based Product Probabilities & 0.9850 & 0.9850 & 0.9850 & $98.11 \%$ \\
SOFT ELA based MIN Probabilities & 0.981 & 0.9835 & 0.9830 & $98.47 \%$ \\
SOFT ELA based MAX Probabilities & 0.9850 & 0.9850 & 0.9850 & $99.14 \%$ \\
Proposed method & & & & \\
Weighted voting ELA & 0.9840 & 0.9841 & 0.9882 & $99.21 \%$ \\
\hline
\end{tabular}

Table 5: The confusion matrix using the weighted voting mechanism-based combined models for 38 classes

\begin{tabular}{|c|c|c|c|c|c|c|c|c|c|c|c|c|c|c|c|c|c|c|c|}
\hline & PBBS & $\mathrm{PH}$ & PLB & PEB & $\mathrm{RH}$ & $\mathrm{SH}$ & SPM & STH & SLS & TTS & TTMV & TTYLCV & TBS & TEB & $\mathrm{TH}$ & TLB & TLM & TSLP & TSMTSSm \\
\hline$\overline{\mathrm{PBBS}}$ & 720 & 0 & 36 & 0 & 0 & 0 & 0 & 0 & 0 & 0 & 25 & 0 & 0 & 0 & 0 & 0 & 0 & 0 & 0 \\
\hline PH & 0 & 116 & 0 & 0 & 0 & 0 & 0 & 0 & 0 & 0 & 0 & 0 & 0 & 0 & 0 & 0 & 0 & 0 & 0 \\
\hline PLB & 0 & 0 & 637 & 0 & 0 & 0 & 0 & 0 & 0 & 37 & 94 & 0 & 0 & 0 & 0 & 0 & 0 & 0 & 0 \\
\hline PEB & 0 & 0 & 0 & 824 & 0 & 0 & 0 & 0 & 0 & 0 & 0 & 0 & 0 & 0 & 0 & 0 & 0 & 0 & 0 \\
\hline RH & 0 & 0 & 0 & 0 & 208 & 0 & 0 & 0 & 0 & 0 & 0 & 0 & 0 & 0 & 0 & 0 & 0 & 0 & 0 \\
\hline $\mathrm{SH}$ & 0 & 1 & 0 & 0 & 0 & 4102 & 0 & 0 & 0 & 0 & 100 & 0 & 0 & 0 & 0 & 0 & 0 & 0 & 0 \\
\hline SPM & 0 & 0 & 0 & 0 & 0 & 0 & 1000 & 0 & 0 & 0 & 490 & 0 & 13 & 0 & 0 & 0 & 0 & 0 & 0 \\
\hline STH & 0 & 0 & 0 & 0 & 0 & 0 & 0 & 287 & 0 & 0 & 53 & 0 & 0 & 48 & 0 & 0 & 0 & 0 & 0 \\
\hline SLS & 0 & 0 & 0 & 0 & 0 & 0 & 0 & 0 & 931 & 0 & 0 & 0 & 0 & 0 & 0 & 0 & 0 & 0 & 0 \\
\hline TTS & 0 & 0 & 0 & 0 & 0 & 0 & 0 & 0 & 0 & 1121 & 15 & 0 & 0 & 0 & 0 & 0 & 0 & 0 & 0 \\
\hline TTMV & 0 & 0 & 0 & 0 & 0 & 0 & 0 & 0 & 0 & 0 & 307 & 0 & 0 & 0 & 0 & 0 & 0 & 0 & 0 \\
\hline TTYLCV & 0 & 0 & 0 & 0 & 0 & 0 & 0 & 0 & 0 & 0 & 12 & 221 & 0 & 0 & 0 & 0 & 0 & 0 & 0 \\
\hline TBS & 0 & 0 & 0 & 0 & 0 & 0 & 0 & 0 & 0 & 0 & 10 & 0 & 494 & 0 & 0 & 0 & 0 & 0 & 0 \\
\hline TEB & 0 & 0 & 0 & 0 & 0 & 0 & 0 & 0 & 0 & 0 & 3 & 0 & 0 & 343 & 0 & 0 & 0 & 0 & 0 \\
\hline $\mathrm{TH}$ & 0 & 0 & 0 & 0 & 0 & 0 & 0 & 0 & 0 & 0 & 4 & 0 & 0 & 0 & 299 & 0 & 0 & 0 & 0 \\
\hline TLB & 0 & 0 & 0 & 0 & 0 & 18 & 0 & 0 & 0 & 0 & 12 & 30 & 0 & 0 & 0 & 1500 & 0 & 0 & 0 \\
\hline TLM & 0 & 0 & 0 & 0 & 0 & 0 & 0 & 0 & 0 & 0 & 0 & 0 & 0 & 0 & 0 & 0 & 768 & 0 & 0 \\
\hline TSLP & 0 & 0 & 0 & 0 & 0 & 0 & 0 & 0 & 0 & 0 & 0 & 0 & 0 & 0 & 0 & 0 & 0 & 1456 & 0 \\
\hline TSMTSSm & 0 & 0 & 0 & 0 & 0 & 0 & 0 & 0 & 0 & 0 & 0 & 0 & 0 & 0 & 0 & 0 & 0 & 0 & 1312 \\
\hline
\end{tabular}

Table 6: The confusion matrix using the weighted voting mechanism-based combined models for 38 classes (continue)

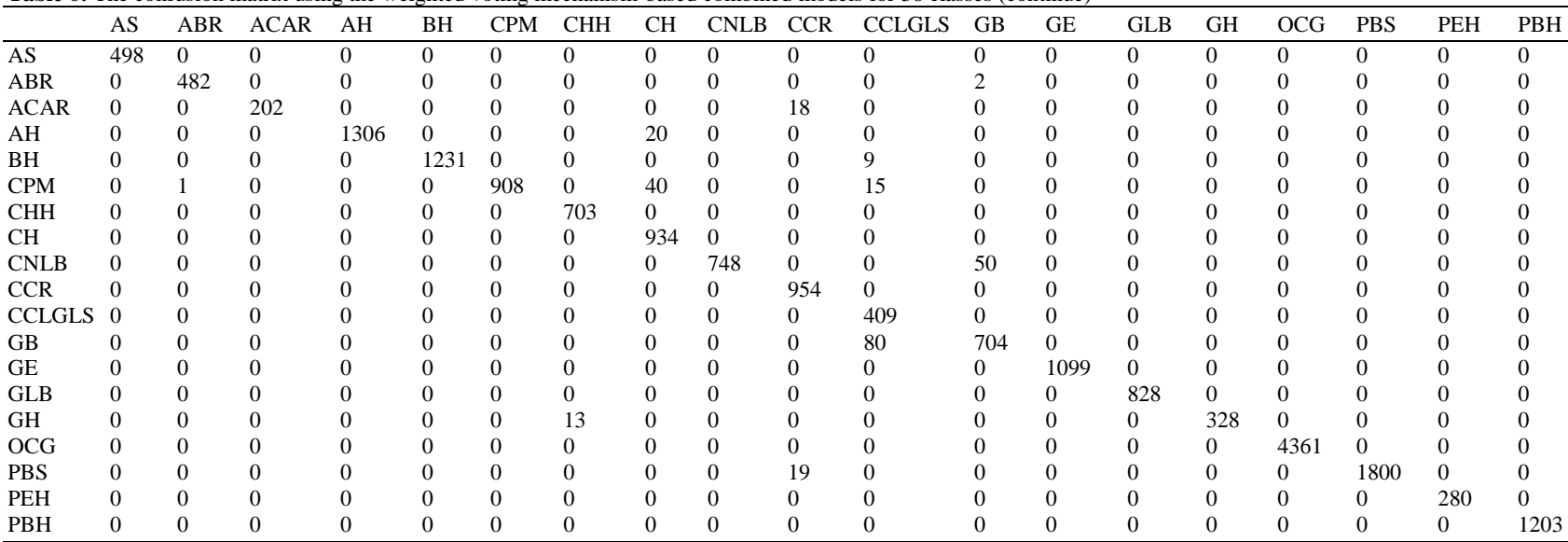

\section{Comparison with Existing Works}

To verify the performance of the proposed technique, 5 methods are selected for comparison in this study. The Fig. 14. displays a comparison of prediction accuracy between the proposed Ensembles deep learning-based the weighted voting mechanism with existing work on plant leaves diseases using the Plant village dataset.

In (Sladojevic et al., 2016) used deep CNN to detect plant diseases from leaf images. This method can distinguish between healthy leaves and some dissimilar diseases. The CNN's whole accuracy is $94.60 \%$. In 
addition, the authors (Wang et al., 2017) used also the CNN method with 50 hidden layers using Neuroph Studio framework as an IDE to build a more facilitated deep Convolutional Neural Network (CNN) whereby the convolution and pooling feature extractions were embedded in the Neuroph library. In this study, the authors found the proposed CNN gave an overall accuracy of $92.85 \%$. On the other hand, in (Ennouni et al., 2021b) proposed a DL approach to estimate disease severity. The best model was trained with transfer learning, which yields an overall accuracy of $90.4 \%$. Comparing with other work, authors in (Ennouni et al., 2021c) used machine learning approach based PDE; this study reported an average classification accuracy of $95.9 \%$.

As you see our proposed method which EL-based weighted voting architectures provided an overall accuracy of $99.21 \%$ and proved its feasibility. As far as we can ascertain, our proposed is performs better than any in the literature to date.

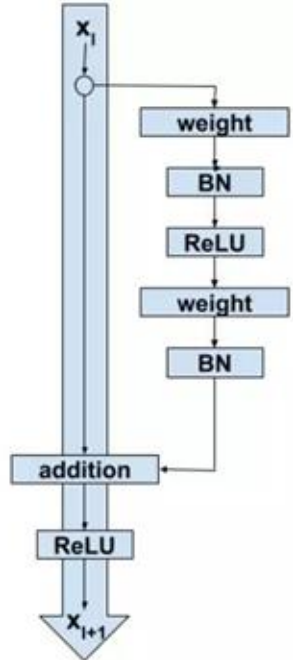

(a)

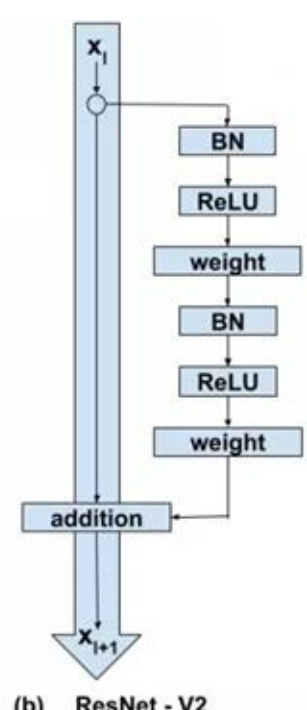

Fig. 8: Res Net Architecture and its versions
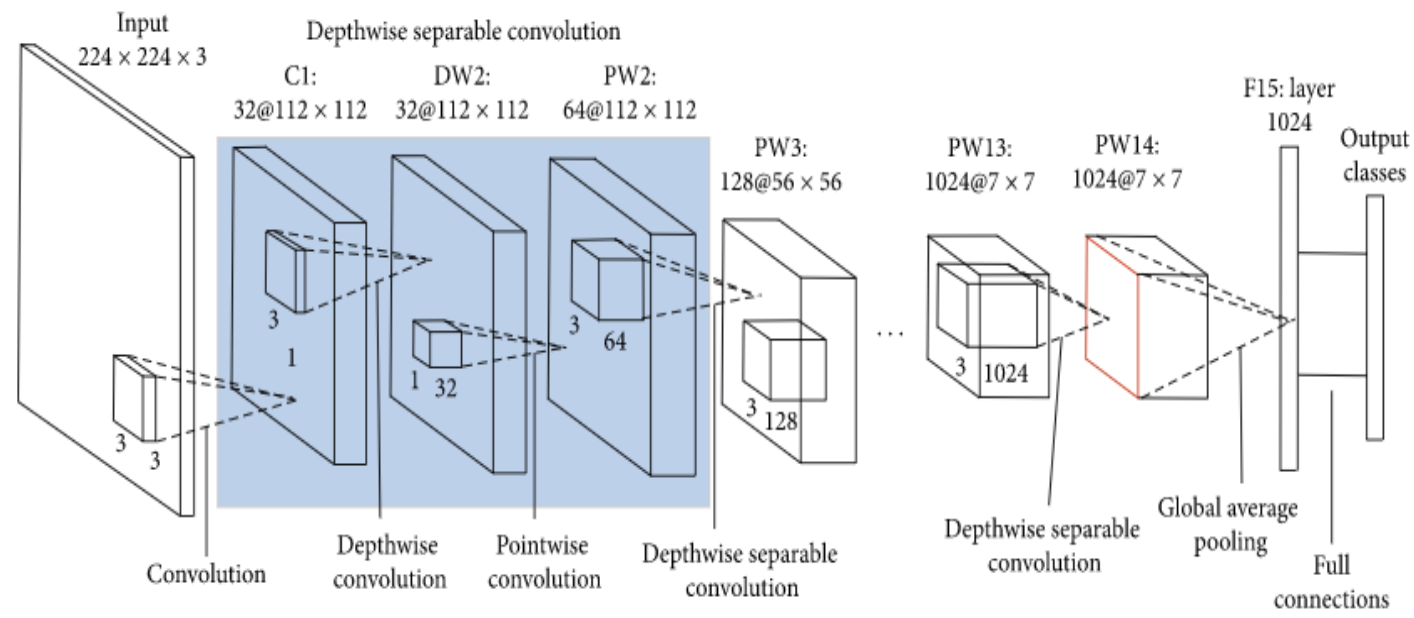

Fig. 9: Mobile Net Architecture

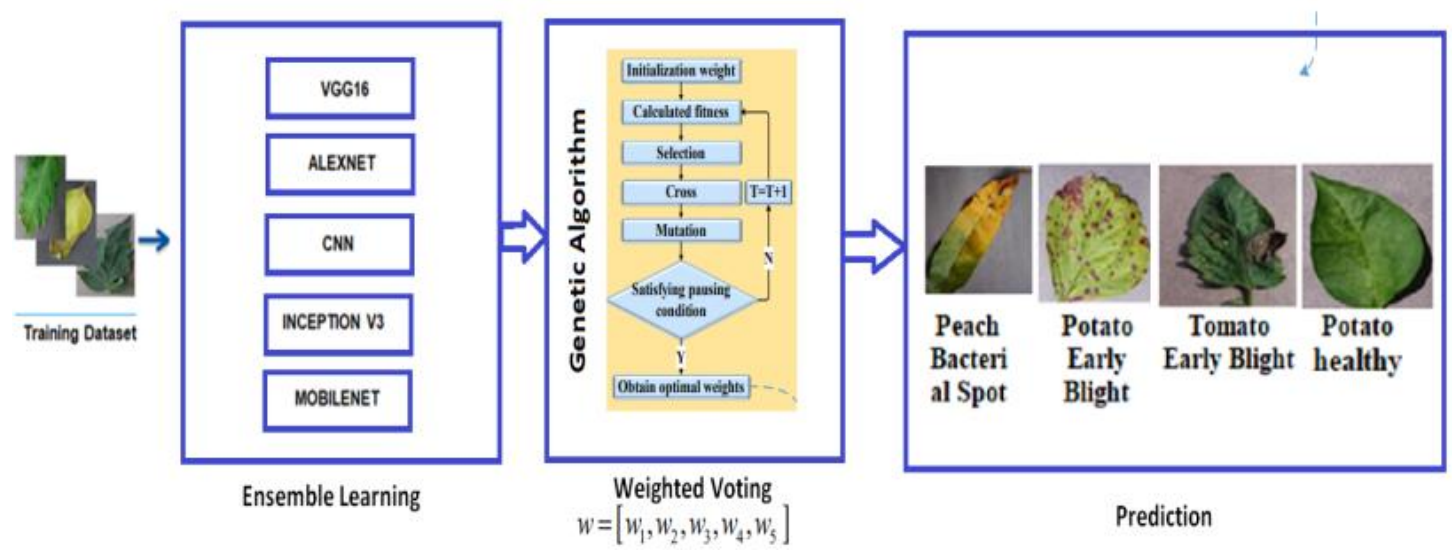

Fig. 10: Overall workflow of proposed weighted voting ensemble model for plant leaf disease classification 


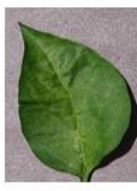

$\mathrm{PBH}$

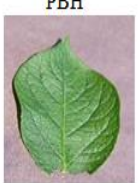

$\mathrm{PH}$

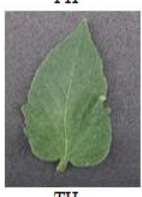

$\mathrm{TH}$

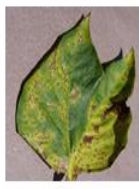

PBB

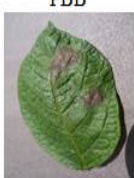

PLB

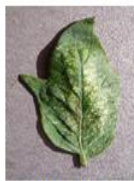

TSMTSSm

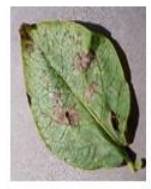

PEB

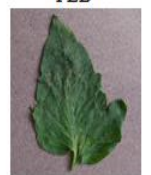

TTS

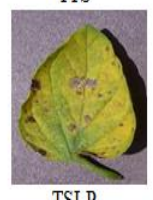

TSLP

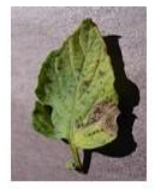

TEB

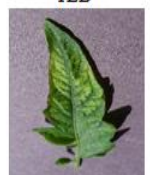

TTMV

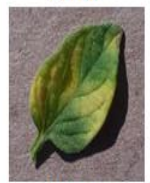

TLM

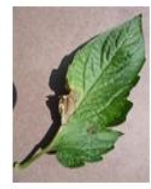

TLB

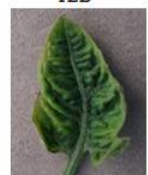

TTYLCV

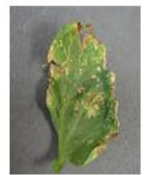

TBS

Fig. 11: Some healthy and diseased plants images from the Plant Village dataset

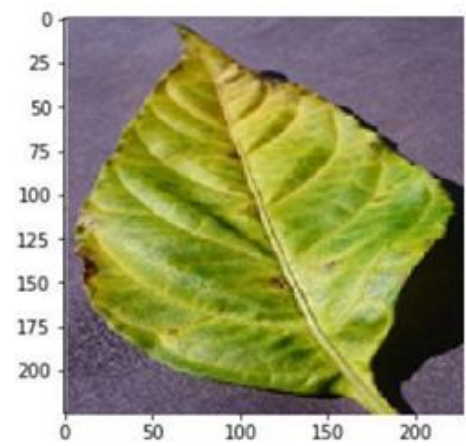

SOURCE : class : Pepper_bell_Bacterial_spot

PREDICTED : class : Pepper_bell_Bacterial_spot

Exactitude : 0.9903

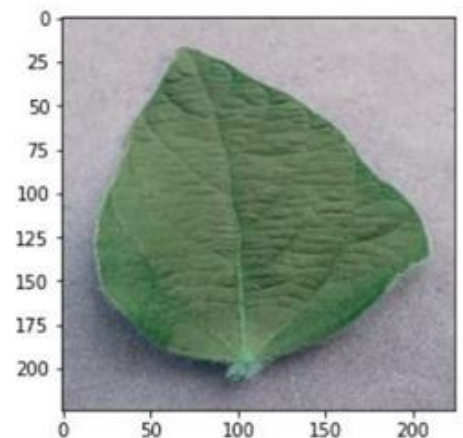

SOURCE : class : Soybean__healthy

PREDICTED : class : Soybean__healthy

Exactitude : 0.9942

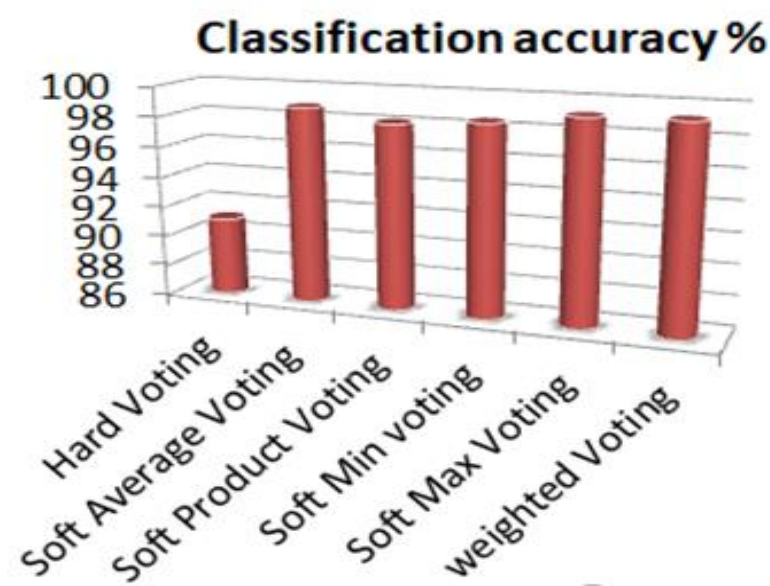

Fig. 12: Classification Accuracy for plant village images dataset

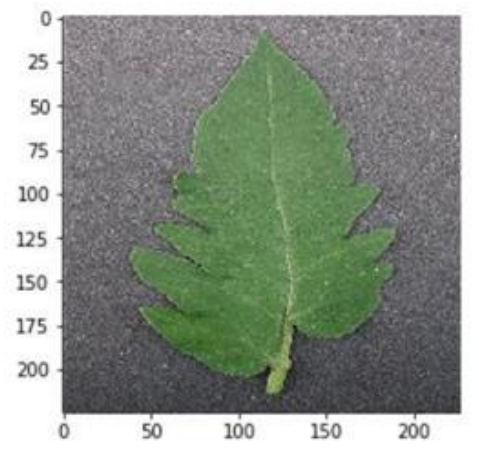

SOURCE : class : Tomato_healthy

PREDICTED : class : Tomato_healthy

Exactitude : 0.9978

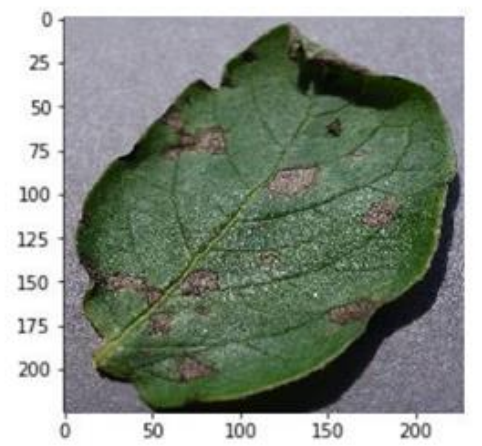

SOURCE : class : Potato__Early_blight

PREDICTED : class : Potato__Early_blight

Exactitude : 0.9946

Fig. 13: Classification accuracy for plant village images 


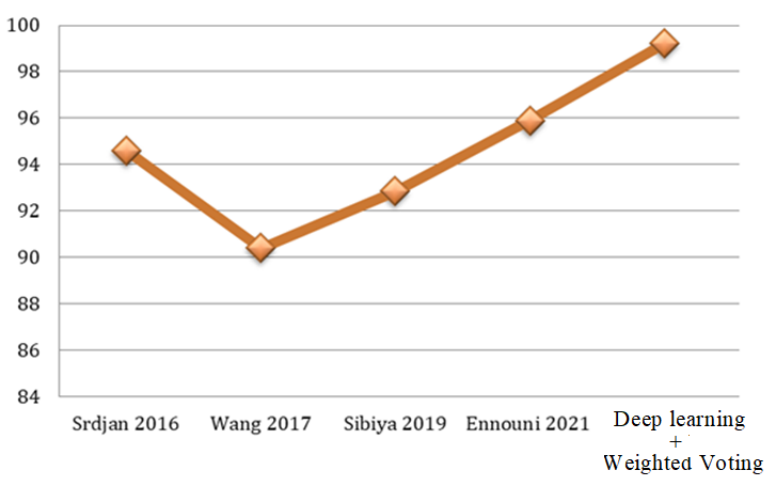

Fig. 14: A comparison of classification accuracy of the proposed method with recent approaches

\section{Conclusion}

This study proposes and implements a weighted voting technique for plant disease detection and classification based on an Ensemble Learning approach. The main contribution is to improve classification accuracy by using a weighted voting mechanism based on a Genetic algorithm to propose the optimal weights. The obtained results prove the effectiveness of our proposed method by giving an average accuracy of $99.21 \%$ on the plant village dataset. We can conclude that this approach can be used to monitor the quality and quantity of plant production. In future work, we intend to deploy this technique on realworld applications to monitor, detect and classify the immense range of plant diseases automatically.

\section{Acknowledgment}

We would like to express our gratitude and thank you to the LISAC laboratory, the Faculty of Sciences, the University Sidi Mohamed Ben Abdellah, Fez, Morocco for the funding and those who are involved directly or indirectly in this research.

\section{Author's Contributions}

Assia Ennouni: Developpement, experimentations testing, validation and writing the manuscript.

Noura Ouled Sihamman: Developpement, experimentations testing.

My Abdelouahed Sabri: Concept developpement, experimentations testing, validation and proof reading.

Abdellah Aarab: Concept developpement, experimentations validation and proof reading.

All authors read and approved the final manuscript.

\section{Ethics}

All information provided in this study is confidential and unique. This paper has neither been published nor is under review elsewhere. There are no ethical issues associated with this research.

\section{References}

Abdelhafiz, D., Yang, C., Ammar, R., \& Nabavi, S. (2019). Deep convolutional neural networks for mammography: Advances, challenges and applications. BMC bioinformatics, 20(11), 1-20. doi.org/10.1186/s12859-019-2823-4

Barbedo, J. G. A. (2013). Digital image processing techniques for detecting, quantifying and classifying plant diseases. SpringerPlus, 2(1), 1-12. https://springerplus.springeropen.com/articles/10.11 86/2193-1801-2-660

Biswas, M., Kuppili, V., Saba, L., Edla, D. R., Suri, H. S., Cuadrado-Godia, E., ... \& Suri, J. S. (2019). State-ofthe-art review on deep learning in medical imaging. Front Biosci (Landmark Ed), 24, 392-426. doi.org/10.2741/4725

Brahimi, M., Boukhalfa, K., \& Moussaoui, A. (2017). Deep learning for tomato diseases: Classification and symptoms visualization. Applied Artificial Intelligence, 31(4), 299-315. doi.org/10.1080/08839514.2017.1315516

Breiman, L. (1996). Bagging predictors. Machine learning, 24(2), 123-140. doi.org/10.1007/BF00058655

Cao, J., Kwong, S., Wang, R., Li, X., Li, K., \& Kong, X. (2015). Class-specific soft voting based multiple extreme learning machines ensemble. Neurocomputing, 149, 275-284. doi.org/10.1016/j.neucom.2014.02.072

Chen, Y., Wong, M. L., \& Li, H. (2014). Applying Ant Colony Optimization to configuring stacking ensembles for data mining. Expert systems with applications, 41(6), 2688-2702. doi.org/10.1016/j.eswa.2013.10.063

Delgado, J., \& Ishii, N. (1999, August). Memory-based weighted majority prediction. In SIGIR Workshop Recomm. Syst. Citeseer (p. 85).

Du, K. L., \& Swamy, M. N. S. (2019). Combining Multiple Learners: Data Fusion and Ensemble Learning. In Neural Networks and Statistical Learning (pp. 737-767). Springer, London. doi.org/10.1007/978-1-4471-7452-3_25

El-Sayed, A. M. A., Rida, S. Z., \& Gaber, Y. A. (2020). Dynamical of curative and preventive treatments in a two-stage plant disease model of fractional order. Chaos, Solitons \& Fractals, 137, 109879. doi.org/10.1016/j.chaos.2020.109879

Ennouni, A., Sabri, M. A., \& Aarab, A. (2021a). Plant Diseases Detection and Classification Based on Image Processing and Machine Learning. In Intelligent Systems in Big Data, Semantic Web and Machine Learning (pp. 283-290). Springer, Cham. doi.org/10.1007/978-3-030-72588-4_20 
Ennouni, A., Sihamman, N. O., Sabri, M. A. \& Aarab, A. (2021a). Early Detection and Classification Approach for Plant Diseases based on MultiScale Image Decomposition. Journal of Computer Science, 17(3), 284-295. doi.org/10.3844/jcssp.2021.284.295.

Ennouni, A., Sihamman, N. O., Sabri, M. A., \& Aarab, A. (2021c, January). Analysis and Classification of Plant Diseases Based on Deep Learning. In International Conference on Digital Technologies and Applications (pp. 123-133). Springer, Cham. doi.org/10.1007/978-3-030-73882-2_12

Ferentinos, K. P. (2018). Deep learning models for plant disease detection and diagnosis. Computers and Electronics in Agriculture, 145, 311-318. doi.org/10.1016/j.compag.2018.01.009

Filali, Y., Khoukhi, H. E., Sabri, M. A., \& Aarab, A. (2020). Efficient fusion of handcrafted and pre-trained CNNs features to classify melanoma skin cancer. Multimedia Tools and Applications, 79(41), 3121931238. doi.org/10.1007/s11042-020-09637-4

Fina, F., Birch, P., Young, R., Obu, J., Faithpraise, B., \& Chatwin, C. (2013). Automatic plant pest detection and recognition using k-means clustering algorithm and correspondence filters. International Journal of Advanced Biotechnology and Research, 4(2), 189-199. https://core.ac.uk/download/pdf/30608241.pdf

Fuentes, A., Yoon, S., Kim, S. C., \& Park, D. S. (2017). A robust deep-learning-based detector for real-time tomato plant diseases and pests recognition. Sensors, 17(9), 2022. doi.org/10.3390/s17092022

He, K., Zhang, X., Ren, S., \& Sun, J. (2016). Deep residual learning for image recognition. In Proceedings of the IEEE conference on computer vision and pattern recognition (pp. 770-778). doi.org/10.1007/s11042-017-4440-4

Szegedy, C., Vanhoucke, V., Ioffe, S., Shlens, J., \& Wojna, Z. (2016). Rethinking the inception architecture for computer vision. In Proceedings of the IEEE conference on computer vision and pattern recognition (pp. 2818-2826).

Howard, A. G., Zhu, M., Chen, B., Kalenichenko, D., Wang, W., Weyand, T., ... \& Adam, H. (2017). Mobilenets: Efficient convolutional neural networks for mobile vision applications. arXiv preprint arXiv:1704.04861.

Huang, K. Y. (2007). Application of artificial neural network for detecting Phalaenopsis seedling diseases using color and texture features. Comput. Electron. Agric. 57, 3-11. doi.org/10.1016/j.compag.2007.01.015

Hughes, D., \& Salathé, M. (2015). An open access repository of images on plant health to enable the development of mobile disease diagnostics. arXiv preprint arXiv:1511.08060. https://arxiv.org/abs/1511.08060
Kang, X. B., Lin, G. F., Chen, Y. J., Zhao, F., Zhang, E. H., \& Jing, C. N. (2020). Robust and secure zerowatermarking algorithm for color images based on majority voting pattern and hyper-chaotic encryption. Multimedia Tools and Applications, 79(1), 1169-1202. doi.org/10.1007/s11042-019-08191-y

Krizhevsky, A., Sutskever, I., \& Hinton, G. E. (2012). Imagenet classification with deep convolutional neural networks. Advances in neural information processing systems, 25, 1097-1105. doi.org/10.1109/CVPR.2015.7298594

Kuncheva, L. I., \& Rodríguez, J. J. (2014). A weighted voting framework for classifiers ensembles. Knowledge and Information Systems, 38(2), 259-275. doi.org/10.1007/s10115-012-0586-6

LeCun, Y., Bengio, Y., \& Hinton, G. (2015). Deep learning. nature, 521(7553), 436-444. doi.org/10.1038/nature14539

LeCun, Y., Boser, B., Denker, J. S., Henderson, D., Howard, R. E., Hubbard, W., \& Jackel, L. D. (1989). Backpropagation applied to handwritten zip code recognition. Neural computation, 1(4), 541-551. doi.org/10.1162/neco.1989.1.4.541

LeCun, Y., Bottou, L., Bengio, Y., \& Haffner, P. (1998). Gradient-based learning applied to document recognition. Proceedings of the IEEE, 86(11), 2278-2324. doi.org/10.1109/5.726791

Litjens, G., Kooi, T., Bejnordi, B. E., Setio, A. A. A., Ciompi, F., Ghafoorian, M., ... \& Sánchez, C. I. (2017). A survey on deep learning in medical image analysis. Medical image analysis, 42, 60-88. doi.org/10.1016/j.media.2017.07.005

Lundervold, A. S., \& Lundervold, A. (2019). An overview of deep learning in medical imaging focusing on MRI. Zeitschrift für Medizinische Physik, 29(2), 102-127. doi.org/10.1016/j.zemedi.2018.11.002

Mathews, D. M. (2010, October). Optimizing detection and management of virus diseases of plants. In Proceedings of the Landscape Disease Symposium (pp. 10-20). https://ucanr.edu/sites/ucnfa/files/62197.pdf

Mohanty, S. P., Hughes, D. P., \& Salathé, M. (2016). Using deep learning for image-based plant disease detection. Frontiers in plant science, 7, 1419. doi.org/10.3389/fpls.2016.01419

Onan, A., Korukoğlu, S., \& Bulut, H. (2016). A multiobjective weighted voting ensemble classifier based on differential evolution algorithm for text sentiment classification. Expert Systems with Applications, 62, 1-16.3 doi.org/10.1016/j.eswa.2016.06.005

Oppenheim, D., Shani, G., Erlich, O., \& Tsror, L. (2019). Using deep learning for image-based potato tuber disease detection. Phytopathology, 109(6), 1083-1087. doi.org/10.1094/PHYTO-08-18-0288-R 
Park, H., Eun, J. S. \& Kim, S. H. (2017). Image-based disease diagnosing and predicting of the crops through the deep learning mechanism, In Information and Communication Technology Convergence (ICTC), IEEE 2017 International Conference on, pp. 129-131. doi.org/10.1109/ICTC.2017.8190957

Petropoulos, F., Hyndman, R. J., \& Bergmeir, C. (2018). Exploring the sources of uncertainty: Why does bagging for time series forecasting work?. European Journal of Operational Research, 268(2), 545-554. doi.org/10.1016/j.ejor.2018.01.045

Phadikar, S., \& Sil, J. (2008, December). Rice disease identification using pattern recognition techniques. In 2008 11th International Conference on Computer and Information Technology (pp. 420-423). IEEE. doi.org/10.1109/ICCITECHN.2008.4803079

Sahni, V., Srivastava, S., \& Khan, R. (2021). Modelling Techniques to Improve the Quality of Food Using Artificial Intelligence. Journal of Food Quality, 2021. doi.org/10.1155/2021/2140010

Sandler, M., Howard, A., Zhu, M., Zhmoginov, A., \& Chen, L. C. (2018). Mobilenetv2: Inverted residuals and linear bottlenecks. In Proceedings of the IEEE conference on computer vision and pattern recognition (pp. 4510-4520). doi.org/10.1109/CVPR.2018.00474

Sibiya, M., \& Sumbwanyambe, M. (2019). A computational procedure for the recognition and classification of maize leaf diseases out of healthy leaves using convolutional neural networks. AgriEngineering, 1(1), 119-131. doi.org/10.3390/agriengineering1010009

Simonyan, K., \& Zisserman, A. (2014). Very deep convolutional networks for large-scale image recognition. arXiv preprint arXiv:1409.1556. https://arxiv.org/abs/1409.1556

Sladojevic, S., Arsenovic, M. anderla, A., Culibrk, D., \& Stefanovic, D. (2016). Deep neural networks based recognition of plant diseases by leaf image classification. Computational intelligence and neuroscience, 2016. doi.org/10.1155/2016/3289801

Szegedy, C., Liu, W., Jia, Y., Sermanet, P., Reed, S., Anguelov, D., ... \& Rabinovich, A. (2015). Going deeper with convolutions. In Proceedings of the IEEE conference on computer vision and pattern recognition (pp. 1-9). doi.org/10.1109/CVPR.2015.7298594

Too, E. C., Yujian, L., Njuki, S., \& Yingchun, L. (2019). A comparative study of fine-tuning deep learning models for plant disease identification. Computers and Electronics in Agriculture, 161, 272-279. doi.org/10.1016/j.compag.2018.03.032
Türkoğlu, M., \& Hanbay, D. (2019). Plant disease and pest detection using deep learning-based features. Turkish Journal of Electrical Engineering \& Computer Sciences, 27(3), 1636-1651. doi.org/10.3906/elk-1809-181

Tyagi, N., Khan, R., Chauhan, N., Singhal, A., \& Ojha, J. (2021). E-Rickshaws Management for Small Scale Farmers using Big Data-Apache Spark. In IOP Conference Series: Materials Science and Engineering (Vol. 1022, No. 1, p. 012023). IOP Publishing. doi.org/10.1088/1757-899X/1022/1/012023

Vijai, S., Namita, S., \& Shikha, S. (2020). A review of imaging techniques for plant disease detection, Artificial Intelligence in Agriculture, Volume 4, Pages 229-242. doi.org/10.1016/j.aiia.2020.10.002

Wang, G., \& Ma, J. (2011). Study of corporate credit risk prediction based on integrating boosting and random subspace. Expert Systems with Applications, 38(11), 13871-13878. doi.org/10.1016/j.eswa.2011.04.191

Wang, G., Sun, Y., \& Wang, J. (2017). Automatic imagebased plant disease severity estimation using deep learning. Computational intelligence and neuroscience, 2017. doi.org/10.1155/2017/2917536

Wolpert, D. H. (2011). Stacked generalization, Neural Networks, vol. 5, no. 2, pp, 241-259. doi.org/10.1016/S0893-6080(05)80023-1

Xiao, Y., Wu, J., Lin, Z., \& Zhao, X. (2018). A deep learning-based multi-model ensemble method for cancer prediction. Computer methods and programs in biomedicine, 153, 1-9. doi.org/10.1016/j.cmpb.2017.09.005

Xu, G., Zhang, F., Shah, S. G., Ye, Y., \& Mao, H. (2011). Use of leaf color images to identify nitrogen and potassium deficient tomatoes. Pattern Recognition Letters, 32(11), 1584-1590. doi.org/10.1016/j.patrec.2011.04.020

Zhang, K., Wu, Q., Liu, A., \& Meng, X. (2018). Can deep learning identify tomato leaf disease? Advances in Multimedia, 2018. doi.org/10.1155/2018/6710865

Zhou, Z. H. (2009). Ensemble learning, Encyclopedia of Biometrics. doi.org/10.1007/978-0-387-73003-5_293 